\title{
Temperature Field Analysis for ZnO Thin-Film Pyroelectric Devices with Partially Covered Electrode
}

\author{
Chun-Ching Hsiao ${ }^{1, *}$, Sheng-Wen Huang ${ }^{1}$ and Rwei-Ching Chang ${ }^{2}$ \\ 'Department of Mechanical Design Engineering, National Formosa University, \\ No. 64, Wunhua Rd., Huwei Township, Yunlin County 632, Taiwan \\ ${ }^{2}$ Department of Mechanical and Computer-Aided Engineering, St. John's University, \\ 499, Sec. 4, Tam King Road, Tamsui, Taipei 251, Taiwan
}

(Received July 6, 2011; accepted February 3, 2012)

Key words: zinc oxide, pyroelectricity, sensor, temperature variation rate

In this study, a finite element modeling is applied to simulate the temperature field of multilayer $\mathrm{ZnO}$ pyroelectric devices. The results show that alterations to the electrode width to improve the temperature variation rate are more successful when the $\mathrm{ZnO}$ film thickness is reduced. The marked improvement in the temperature variation rate in the $\mathrm{ZnO}$ layer of $200 \mathrm{~nm}$ thickness indicates a saturation rate of about $27 \%$ when the electrode width is approximately $1 \mu \mathrm{m}$. Furthermore, the optimal electrode width is reduced when the $\mathrm{ZnO}$ film thickness is decreased. Decreasing the $\mathrm{ZnO}$ film thickness clearly increases the temperature variation rate and reduces the response time; an electrode with the optimal width further enhances the temperature variation rate. Moreover, the temperature variation rate significantly decreases for thinner $\mathrm{ZnO}$ films when the electrode width is smaller than the optimal value. In addition, an experimental result is successful to verify the simulation results, and the electrode width is a critical parameter for designing a pyroelectric sensor.

\section{Introduction}

$\mathrm{ZnO}$ is a unique material because it possesses such properties as semiconductivity, piezoelectricity, and pyroelectricity. Wide-band-gap wurtzite phase $\mathrm{ZnO}$ has attracted attention owing to its versatility in many promising applications, such as blue and ultraviolet light emitters, transparent conductors, solar cell windows, gas sensors, photovoltaic devices, pyroelectric sensors, surface acoustic wave (SAW) devices, and

*Corresponding author: e-mail: cchsiao@nfu.edu.tw 
film bulk acoustic resonators (FBARs). Pyroelectric devices have been successfully used in many applications, such as pollution monitoring, hot image detectors, intruder alarms, and gas analysis. ${ }^{(1-3)}$ Thin-film pyroelectric sensors have many advantages, such as integration with on-chip circuitry, uncooled detection, room-temperature operation, speed, lower system cost, portability, and wide spectral response with high sensitivity. In addition, pyroelectricity has been applied to environmental energy-harvesting systems. Pyroelectric energy conversion offers a novel and direct way of converting timedependent temperature fluctuations into electricity for micropower generators and lowenergy consumption systems. ${ }^{(4)}$

Thin-film pyroelectric sensors comprise a pyroelectric layer sandwiched between top and bottom electrodes, and built on a thermal-isolation structure to block heat loss. The top side is exposed to a heat source. The principle of thin-film pyroelectric sensors is based on the pyroelectric effect, which converts the temperature variation to the corresponding electrical signal. The dynamic pyroelectric response current of thin-film pyroelectric sensors can be described using eq. (1):

$$
i_{\mathrm{p}}=\eta \times P \times A \times \mathrm{d} T / \mathrm{d} t,
$$

where $\eta$ is the absorption coefficient of radiation, $P$ is the pyroelectric coefficient of the pyroelectric film, $A$ is the sensing area, and $\mathrm{d} T / \mathrm{d} t$ is the temperature variation rate of the pyroelectric film. The thermal-isolation structure, sensitive material properties, top-electrode layout, and absorption coefficient of pyroelectric devices are their most important performance-enhancing qualities. From eq. (1), it can be seen that a higher temperature variation rate in pyroelectric films leads to a higher response current in the pyroelectric sensors. Moreover, a partially covered top electrode has been proven to reveal a higher responsivity than a fully covered electrode because it allows the $\mathrm{ZnO}$ layer to make a direct contact with the heat source. ${ }^{(5)}$ Moreover, a web-type top electrode has been designed to enhance the responsivity of $\mathrm{ZnO}$ pyroelectric sensors by qualitative analysis. ${ }^{(6)}$ A larger piecewise area of the exposed $\mathrm{ZnO}$ layer leads to more heat absorption but, in another aspect, to more dispersed top electrodes. Thus, the design of the web-type top electrode can consider both the piecewise area of the exposed $\mathrm{ZnO}$ layer and the dispersion of top electrodes. The outer regions of the web-type possess a larger piecewise area of the exposed $\mathrm{ZnO}$ layer, while the inner regions possess the lower dispersion of top electrodes. Therefore, the temperature variation rate increases in pyroelectric films when the partially covered top electrode is applied in pyroelectric sensors. This concept has been further expanded to the etching of a three-dimensional pattern on the responsive element of $\mathrm{LiTaO}_{3}$, with lateral temperature gradients induced on the sidewalls of the responsive element under homogeneous irradiation. ${ }^{(7)}$ Thus, the temperature variation rate increases in the responsive element which, in turn, increases the voltage responsivity of pyroelectric sensors. Moreover, the temperature variation rate is difficult to extract from thin films by experimental measurement. One study used a finite element model built using the commercial software ANSYS to explore the temperature variation rate in pyroelectric elements with various thermal properties and geometries. ${ }^{(8)}$ 
The pyroelectricity of $\mathrm{ZnO}$ is attributable to non-centrosymmetrical crystals and so it has a specific polar axis along the direction of spontaneous polarization. ${ }^{(1,2)}$ The most densely packed and thermodynamically favorable growth orientation in a $\mathrm{ZnO}$ wurtzite structure is one in which the c-axis is perpendicular to the substrate. $\mathrm{ZnO}$ films with the c-axis normal to the substrate are preferred in many applications, such as $\mathrm{ZnO}$ pyroelectric devices ${ }^{(5,9)}$ and film bulk acoustic resonators. ${ }^{(10)}$ When $\mathrm{ZnO}$ is subjected to temperature variations, its internal polarization will produce an electric field. Therefore, increasing the responsivity of a $\mathrm{ZnO}$ pyroelectric sensor depends on increasing the temperature variation rate of the $\mathrm{ZnO}$ layer, adopting a $\mathrm{ZnO}$ film with a strongly preferred orientation towards the c-axis, and using a high-performance thermal-isolation structure.

In this paper, our focus was on exploring the temperature variation rate in multilayer $\mathrm{ZnO}$ pyroelectric devices with a partially covered top electrode. The top electrode layout and size were designed and implemented, and a finite element model built using a commercial multiphysics software COMSOL MULTIPHYSICS ${ }^{\circledR} 3.5$ was used to explore the temperature variation rate in multilayer $\mathrm{ZnO}$ pyroelectric devices.

\section{Materials and Methods}

\subsection{Structure and fabrication of pyroelectric sensor}

The present $\mathrm{ZnO}$ thin-film pyroelectric sensor is a sandwich structure composed of a $\mathrm{ZnO}$ sensing layer sandwiched between top and bottom electrodes, and built on a silicon substrate with a thermal-insulation layer to reduce heat loss. The pyroelectric signal is proportional to the temperature variation rate in the $\mathrm{ZnO}$ layer. In other words, a higher temperature variation rate in the $\mathrm{ZnO}$ layer leads to a higher response current for pyroelectric sensors. A partially covered top electrode has a higher responsivity than a fully covered top electrode because the uncovered part of the $\mathrm{ZnO}$ layer is directly exposed to the heat source and, thus, markedly increases the heat absorption. ${ }^{(5)}$ A larger top electrode area reduces the temperature variation rate in the $\mathrm{ZnO}$ layer; however, a larger bare top electrode area increases the temperature variation rate in the $\mathrm{ZnO}$ layer but decreases the responsivity of sensors owing to the smaller top electrode area to hold electrical charges. Therefore, we designed a partially covered top electrode with a mesh type. Figure 1 shows the schematic of the multilayer $\mathrm{ZnO}$ pyroelectric sensor with the fully covered and mesh top electrodes. The layout of the mesh top electrode had the same ratio for the widths of the electrode and bare electrode to deal with both the temperature variation rate and top electrode area. The dimensions of the top electrodes are detailed in Fig. 2. The area of the mesh top electrode was about $12.15 \mathrm{~mm}^{2}$ when the electrode width $(W)$ was fixed at $0.5,1,2,3,4,5,6,10$, and $20 \mu \mathrm{m}$, and the area of the fully covered top electrode was about $14.4 \mathrm{~mm}^{2}$. Moreover, $W$ seemed to be related to the thickness of the $\mathrm{ZnO}$ sensing layer. $W$ could then be optimized by exploring and analyzing the temperature variation rate in $\mathrm{ZnO}$ layers of varying thicknesses.

The fabrication flow of the $\mathrm{ZnO}$ pyroelectric device was divided into several steps, as follows. A silicon wafer with both sides polished was used as a substrate to support

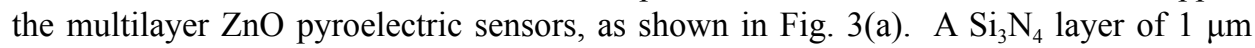




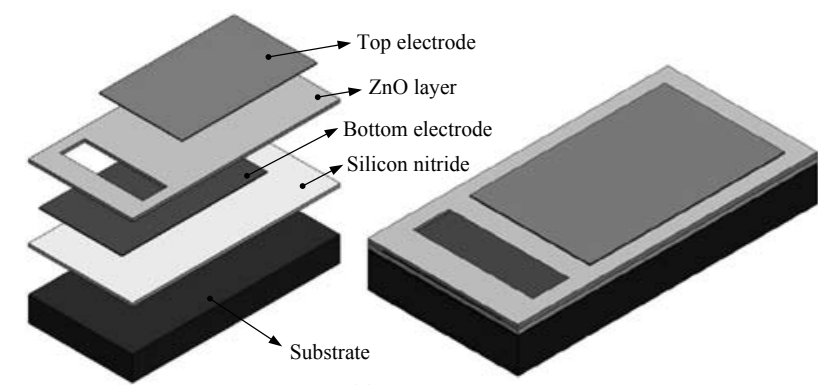

(a)

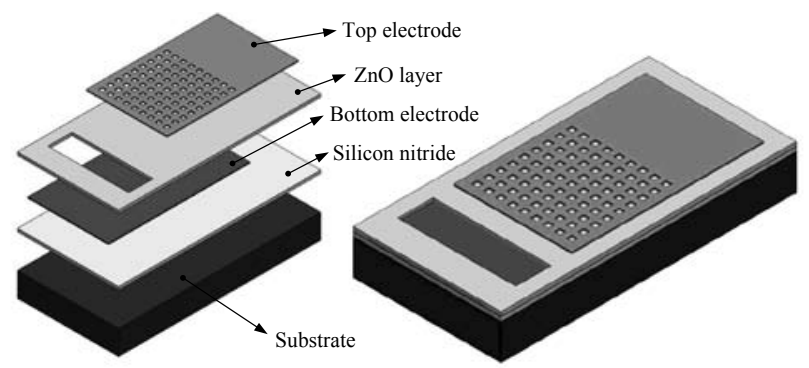

(b)

Substrate $\square$ Silicon nitride

Bottom electrode

ZnO layer $\square$ Top electrode

Fig. 1. Schematic of the multilayer $\mathrm{ZnO}$ pyroelectric sensors: (a) fully covered electrode and (b) mesh top electrode.

thickness was deposited on both sides of the silicon wafer by low-pressure chemical vapor deposition (LPCVD), as shown in Fig. 3(b). The silicon nitride layer can obstruct the thermal conduction to the silicon substrate. The bottom electrode was deposited on the substrates by electron beam evaporation and patterned by wet etching, as shown in Fig. 3(c). The bottom and top electrodes were both composed of gold and chromium. The chromium was an adhesion layer to promote the adhesion between the gold electrode and the substrate or $\mathrm{ZnO}$ films. The thicknesses of gold and chromium were 50 and 5 $\mathrm{nm}$, respectively. The next step was to deposit $\mathrm{ZnO}$ films by sputtering, as shown in Fig. 3(d). A ZnO target with $99.99 \%$ purity was adopted. The $\mathrm{ZnO}$ target was presputtered for $15 \mathrm{~min}$ prior to the film deposition to remove any surface impurity. The chamber was pumped to the base pressure of $8 \times 10^{-7}$ Torr before sputtering. The chamber was then filled with the mixture of argon and oxygen with the gas-mixing ratio of 5:3. The chamber pressure was $2 \times 10^{-3}$ Torr during deposition. The substrate was heated up to $200^{\circ} \mathrm{C}$ during deposition, which was beneficial for improving the $\mathrm{ZnO}$ film quality. The 


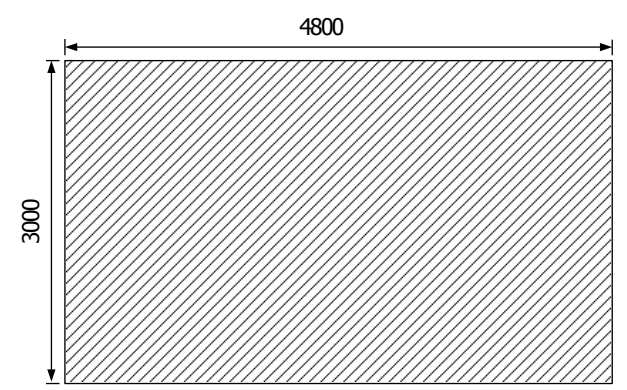

(a)

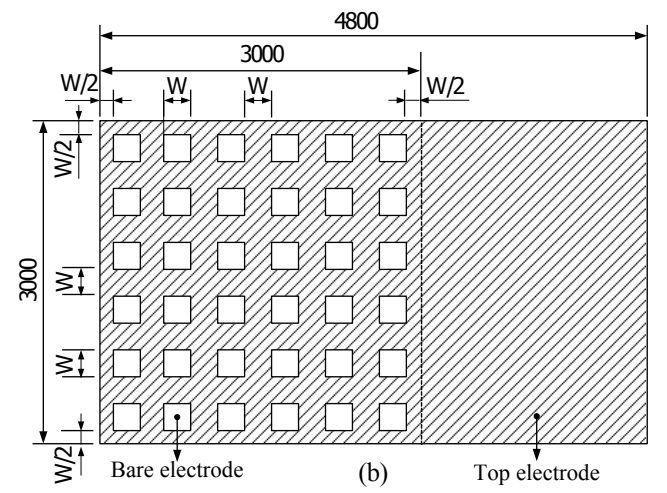

Fig. 2. Top electrode dimensions in micrometer: (a) fully covered type and (b) mesh type.

sputtering RF power significantly affects the $\mathrm{ZnO}$ film quality, and therefore, affects the responsivity of $\mathrm{ZnO}$ pyroelectric sensors. A two-step sputtering process, with a lower RF power of $120 \mathrm{~W}$ followed by a higher RF power of $150 \mathrm{~W}$, was used to achieve a better responsivity of pyroelectric sensors. ${ }^{(9)}$ The first-step RF power was used to deposit onetenth of the required total $\mathrm{ZnO}$ film thickness when using the two-step sputtering process. The processing step in Fig. 3(e) was used to deposit the top electrode on the $\mathrm{ZnO}$ layer. The composition and deposition of the top electrode were similar to those of the bottom electrode, except that the top electrode was patterned by the lift-off method. Finally, as shown in Fig. 3(f), the wet etchant comprising $\mathrm{CH}_{3} \mathrm{COOH}: \mathrm{H}_{3} \mathrm{PO}_{4}: \mathrm{H}_{2} \mathrm{O}=1: 1: 10$ was used to pattern the $\mathrm{ZnO}$ layer to open the bonding pad of the bottom electrode.

\subsection{Finite element model and simulation}

Temperature variation rates in the pyroelectric layers markedly affect the responsivity of pyroelectric sensors. A partially covered electrode has better output signals than a fully covered electrode. ${ }^{(5)}$ Increasing the temperature variation rate or the top electrode area can improve the electrical signals of pyroelectric devices. Therefore, a partially covered mesh-type electrode was used in the $\mathrm{ZnO}$ pyroelectric devices to maximize both 


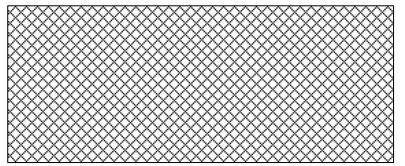

(a)

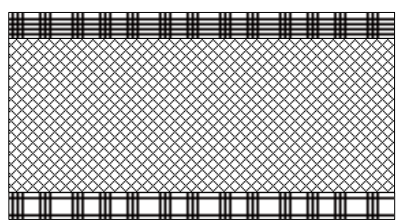

(b)

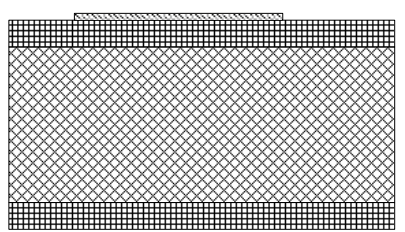

(c)

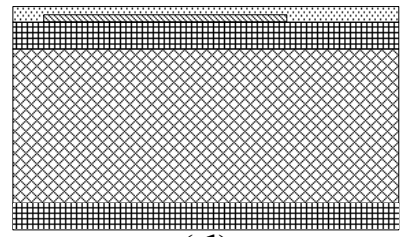

(d)

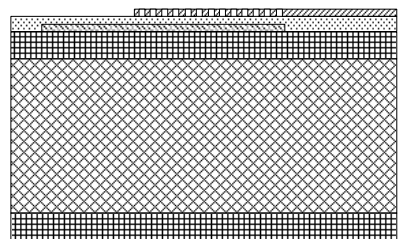

(e)

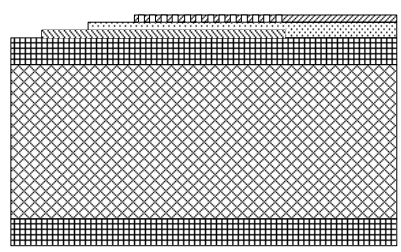

(f)

Silicon substrate

$\mathrm{ZnO}$ layer

Silicon nitride layer Top electrode $(\mathrm{Au})$

Bottom electrode (Au)

Fig. 3. Process flow of multilayer $\mathrm{ZnO}$ pyroelectric sensor with mesh-type top electrode: (a) using silicon wafer as the substrate, (b) depositing $\mathrm{Si}_{3} \mathrm{~N}_{4}$ layers on both sides of the substrate by LPCVD, (c) depositing the bottom electrode by electron beam evaporation and wet etching the pattern, (d) depositing $\mathrm{ZnO}$ layer by $\mathrm{RF}$ magnetron sputtering, (e) depositing top electrode by electron beam evaporation and patterning by lift-off, and (f) patterning the $\mathrm{ZnO}$ layer by wet etching to open the bonding pad of the bottom electrode.

the temperature variation rate and the top electrode area. However, the optimal $W$ in the mesh top electrode seemed to be related to the thickness of the $\mathrm{ZnO}$ sensing layer. In the present study, a two-dimensional finite element model was generated using a commercial multiphysics software COMSOL MULTIPHYSICS ${ }^{\circledR} 3.5$ to explore the temperature variation rate in the multilayer $\mathrm{ZnO}$ pyroelectric sensors with a mesh top electrode as compared with a fully covered top electrode. The optimal $W$ in the mesh top electrode was determined under various $\mathrm{ZnO}$ thicknesses by exploring the temperature variation rate in the $\mathrm{ZnO}$ sensing layer. The material parameters of the films and substrate 
are shown in Table 1. There was an isotropic assumption for the films and substrate properties in this model. The model was meshed using a regular mesh, as shown in Fig. 4. The thicknesses of the $\mathrm{ZnO}$ layers were 200,600 , and $1200 \mathrm{~nm}$. W in the mesh top electrode varied from $0.5,1,2,3,4,5,6,10$ to $20 \mu \mathrm{m}$. The incident irradiation power applied on the top side of the multilayer $\mathrm{ZnO}$ pyroelectric devices was approximately $1.228 \times 10^{-12} \mathrm{~W} / \mu \mathrm{m}^{2}{ }^{\left({ }^{(8)}\right.}$ The thermal isolation condition was applied to the backside of the silicon substrate, and the symmetric condition was applied to the two lateral sides as boundary conditions.

Table 1

Material parameters used for finite element analysis.

\begin{tabular}{lcccc}
\hline Material & $\begin{array}{c}\text { Thermal } \\
\text { conductivity } \\
\left(\mathrm{Wm}^{-1} \mathrm{~K}^{-1}\right)\end{array}$ & $\begin{array}{c}\text { Specific heat } \\
\left(\mathrm{Jg}^{-1} \mathrm{~K}^{-1}\right)\end{array}$ & $\begin{array}{c}\text { Density } \\
\left(\mathrm{gcm}^{-3}\right)\end{array}$ & $\begin{array}{c}\text { Thickness } \\
(\mu \mathrm{m})\end{array}$ \\
\hline Silicon substrate & 163 & 0.703 & 2.33 & 5 \\
Silicon nitride & 20 & 0.700 & 3.10 & 1 \\
Top and bottom electrodes & 317 & 0.129 & 19.3 & 0.1 \\
Zinc oxide & 6 & 0.125 & 5.676 & $0.2,0.6,1.2$ \\
\hline
\end{tabular}

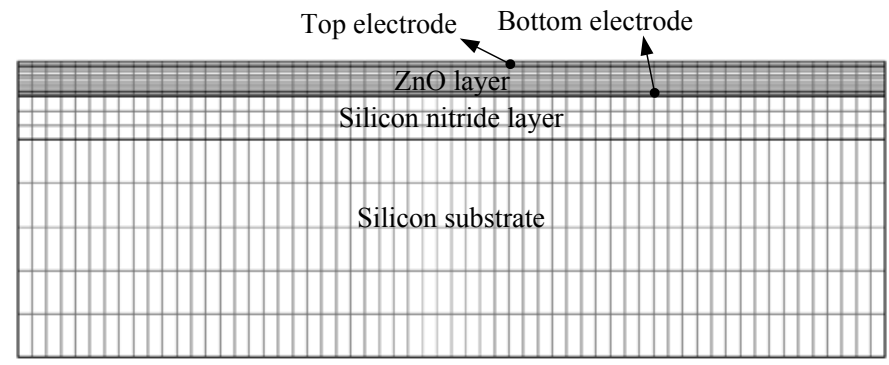

(a)

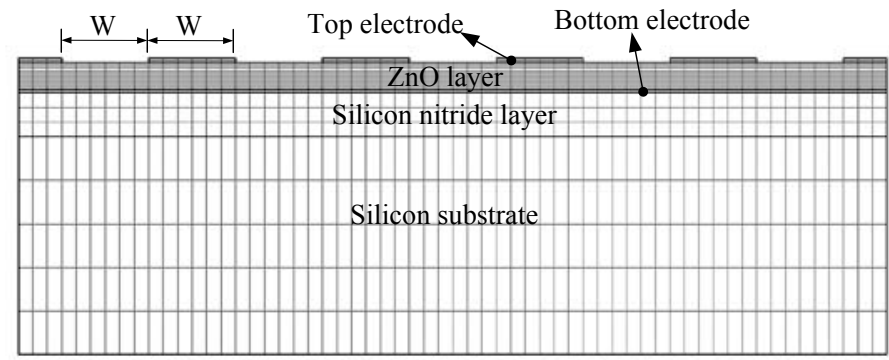

(b)

Fig. 4. Two-dimensional finite element model for $\mathrm{ZnO}$ thin-film pyroelectric devices: (a) fully covered electrode and (b) mesh top electrode. 


\subsection{Signal measurement}

A responsivity measurement system (Fig. 5) was used to evaluate the performance of the present $\mathrm{ZnO}$ pyroelectric sensors. The radiation source was a calibrated infrared (IR) laser of $900 \mathrm{~nm}$ wavelength and $7 \mathrm{~mW}$ maximum power. The power spectrum of the laser was also measured using a power meter by Fourier transform infrared (FTIR) spectroscopy. The laser beam was chopped and molded as a square wave with a modulated frequency $(\omega)$ by a programmable function generator. The modulated beam was split into two beams by a prism. The two beams had the same power, one was reflected on a photodiode as the reference, and the other was expanded via a beam expander such that the beam spot could cover the entire region of the patterned top electrode of the $\mathrm{ZnO}$ pyroelectric sensors. The photodiode was used simply for visual reference on the oscilloscope. The output voltage of the sensor was amplified using an SR560 low-noise voltage amplifier. Both the output signals of the sensors and the photodiode were recorded and displayed using a digital oscilloscope.

\section{Results and Discussion}

When $\mathrm{ZnO}$ is subjected to temperature variation, its internal polarization produces an electrical field that induces voltage responsivity between the top and bottom electrodes. The responsivity is proportional to the temperature variation rate in the $\mathrm{ZnO}$ layer: no

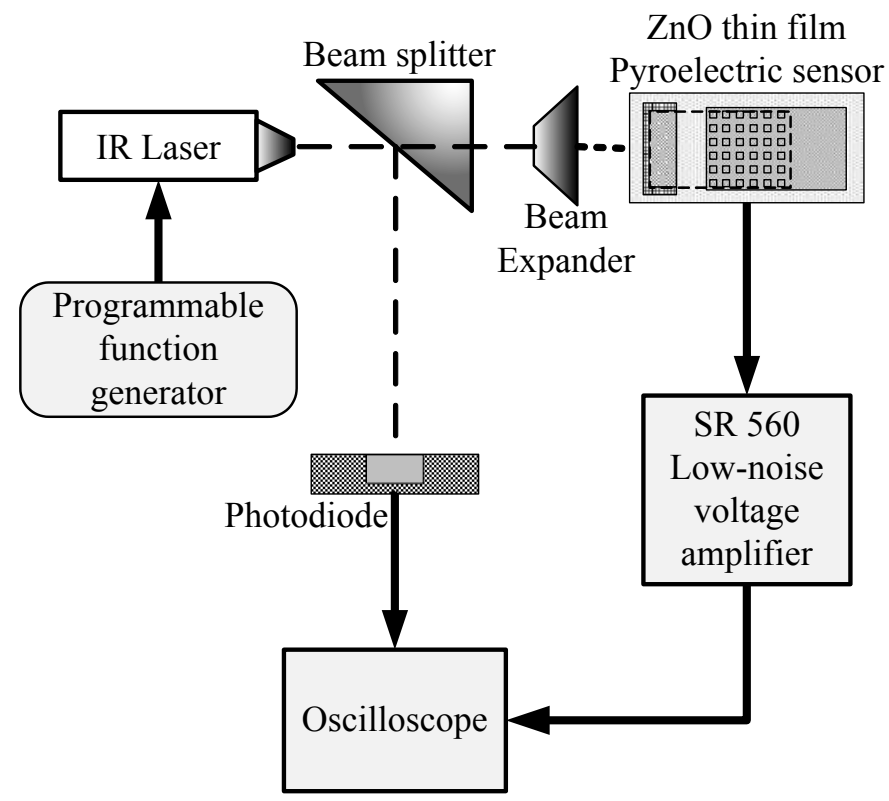

Fig. 5. Schematic diagram of the responsivity measurement experiment. 
temperature variation in the $\mathrm{ZnO}$ layer results in no internal polarization change, and thus, no response voltage. Therefore, the transient temperature fields in multilayer $\mathrm{ZnO}$ pyroelectric sensors were simulated. The points are defined in Fig. 6 and used to explain the temperature variation rate in the $\mathrm{ZnO}$ pyroelectric devices with a mesh top electrode compared with a fully covered top electrode. The points A1, B1, C1, D1, E1, and $\mathrm{F} 1$ were defined at the top of the $\mathrm{ZnO}$ layer. The points $\mathrm{A} 3, \mathrm{~B} 3, \mathrm{C} 3, \mathrm{D} 3, \mathrm{E} 3$, and F3 were defined at the middle of the $\mathrm{ZnO}$ layer. The points A5, B5, C5, D5, E5, and F5 were defined at the bottom of the $\mathrm{ZnO}$ layer. In the mesh top electrode, the temperature variation rates at the points under the electrodes were only useful to produce the electrical charges.

Figure 7 shows the relationship between the temperature variation rate and time in the $\mathrm{ZnO}$ film along the thickness direction from points $\mathrm{A} 1$ to A5, while the fully covered top electrode with a 200 -nm-thick $\mathrm{ZnO}$ layer was used to fabricate the $\mathrm{ZnO}$ pyroelectric sensor. When the point approached the top side of the $\mathrm{ZnO}$ layer, the temperature variation rate further increased, and its maximum peak moved leftward, reducing the response time. Hence, point A5 had the lowest temperature variation rate in the $\mathrm{ZnO}$ layer under the design of the fully covered top electrode. When a mesh top

\begin{tabular}{|llll|}
\hline Fully covered top electrode & & $\bullet$ A 1 \\
\hline ZnO layer & & A 2 \\
& & & \\
\hline Bottom electrode & & & \\
\hline Silicon nitride layer & & \\
\hline Silicon substrate & & \\
\hline
\end{tabular}

(a)

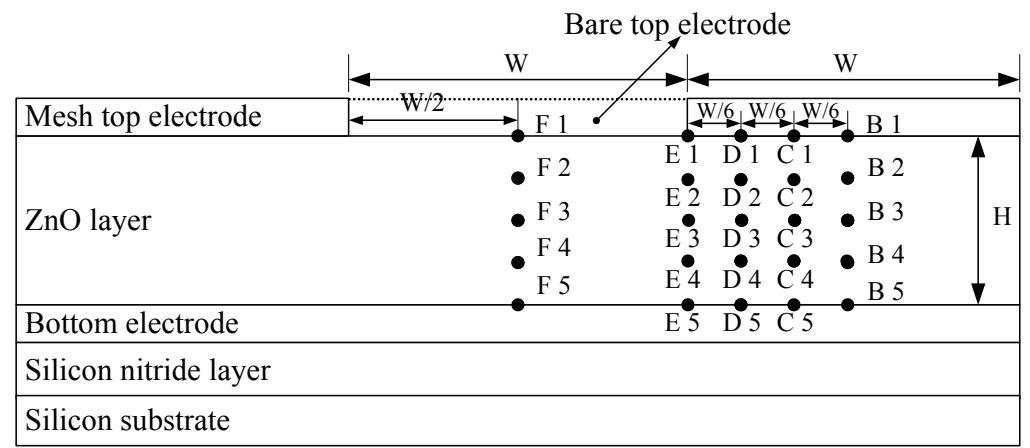

(b)

Fig. 6. Points defined in $\mathrm{ZnO}$ layers: (a) fully covered electrode and (b) mesh top electrode. 


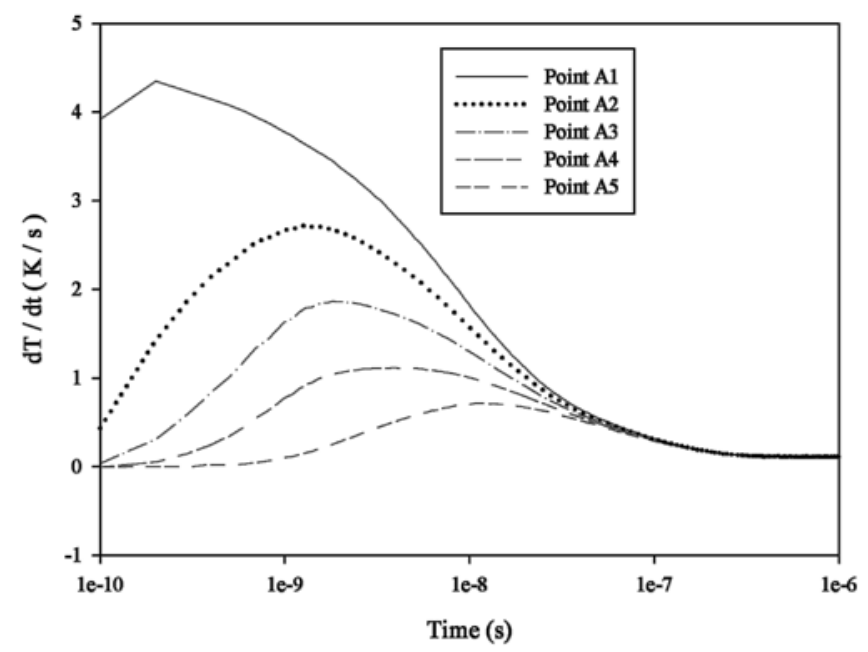

Fig. 7. Relationship between temperature variation rate $(\mathrm{d} T / \mathrm{d} t)$ and time in $\mathrm{ZnO}$ film along the thickness direction in a fully covered electrode with a 200-nm-thick $\mathrm{ZnO}$ layer.

electrode with $W$ of $5 \mu \mathrm{m}$ and a 200 -nm-thick $\mathrm{ZnO}$ layer was used to fabricate the $\mathrm{ZnO}$ pyroelectric sensor, point F1 showed direct contact with the irradiation source. The temperature variation rate at point $\mathrm{F} 1$ was clearly higher than at points $\mathrm{A} 1, \mathrm{~B} 1, \mathrm{C} 1, \mathrm{D} 1$, or E1, as shown in Fig. 8. Moreover, the temperature variation rate at point E1 was higher than at points B1, C1 or D1 because point E1 approached the uncovered top electrode directly to contact the heat source. The temperature variation rate at point E5 was also higher than that at points D5, C5 or B5, as shown in Fig. 9. Therefore, as the point started from the center of the mesh top electrode projected to the $\mathrm{ZnO}$ layer (points B1, B2, B3, B4, or B5) and moved to the edge of the mesh top electrode projected to the $\mathrm{ZnO}$ layer (points E1, E2, E3, E4, or E5), the temperature variation rate further increased and its maximum peak moved leftward. Figure 10 shows the relationship between the temperature variation rate and time in the $\mathrm{ZnO}$ film from points $\mathrm{B} 1$ to $\mathrm{B}$, with the temperature variation rate further increasing and its maximum peak moving leftward when the point approached the top side of the $\mathrm{ZnO}$ layer. Hence, point $\mathrm{B} 5$ had the lowest temperature variation rate in the $\mathrm{ZnO}$ layer under the design of the mesh top electrode. Points at the top side of the $\mathrm{ZnO}$ layer had the largest temperature variation rate, which could hardly be improved. However, improvement in the temperature variation rate at point B5, which possessed the lowest temperature variation rate, certainly enhanced the responsivity of the pyroelectric devices. Figure 11(a) shows the transient temperature variation field at the maximum peak's time of point A5 when the fully covered top electrode with a 200 -nm-thick $\mathrm{ZnO}$ layer was used to fabricate the $\mathrm{ZnO}$ pyroelectric sensor. The temperature variation rate in the $\mathrm{ZnO}$ film increased gradually toward the top electrode owing to the incident radiation power applied on the top electrode. Figure 


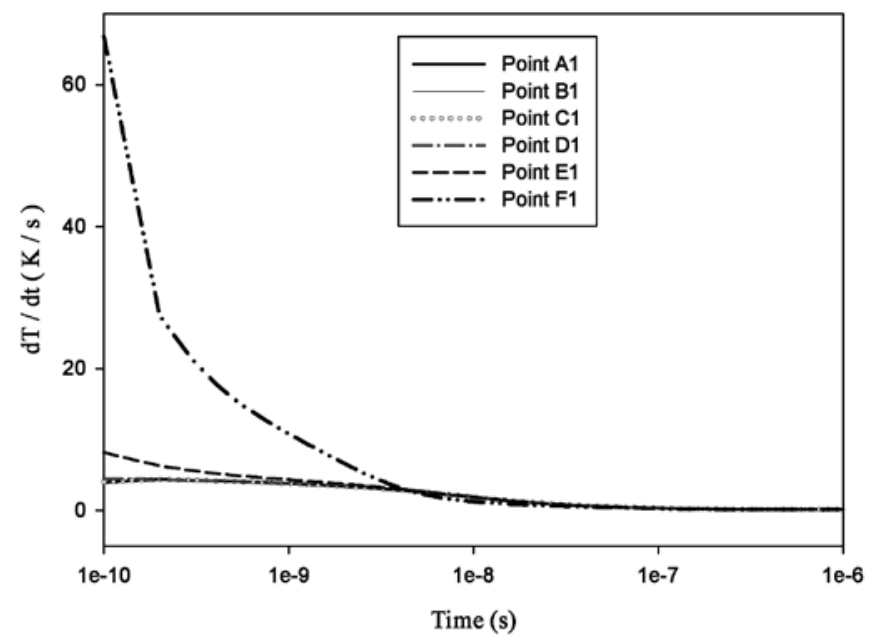

Fig. 8. Relationship between temperature variation rate $(\mathrm{d} T / \mathrm{d} t)$ and time in $\mathrm{ZnO}$ film at points $\mathrm{A} 1$ to F1: a mesh top electrode with a $5 \mu \mathrm{m}$ electrode width and a 200 -nm-thick ZnO layer compared with a fully covered top electrode with a 200 -nm-thick $\mathrm{ZnO}$ layer.

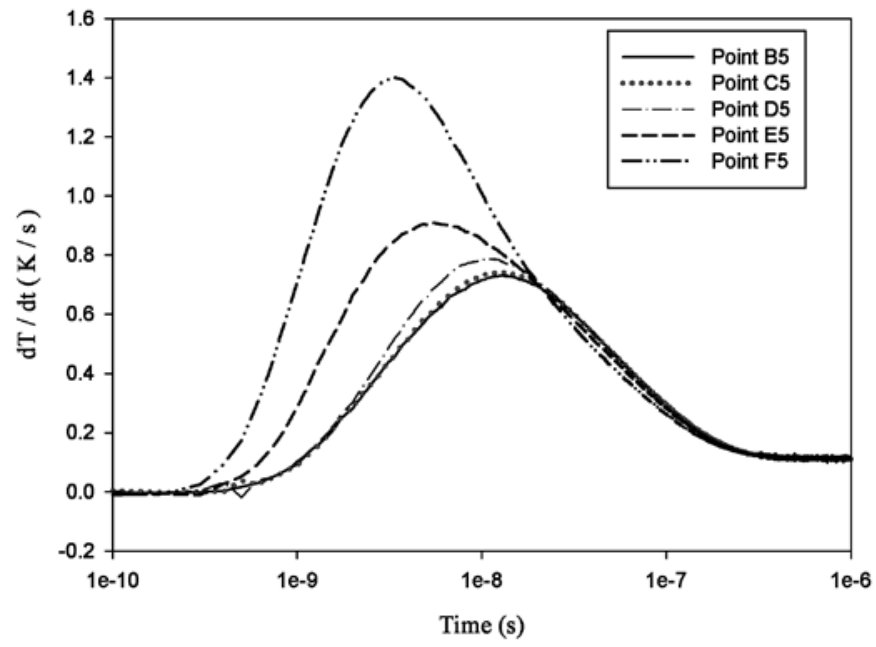

Fig. 9. Relationship between temperature variation rate $(\mathrm{d} T / \mathrm{d} t)$ and time in $\mathrm{ZnO}$ film at points B5 to F5 for a mesh top electrode with a $5 \mu \mathrm{m}$ electrode width and a 200 -nm-thick ZnO layer. 


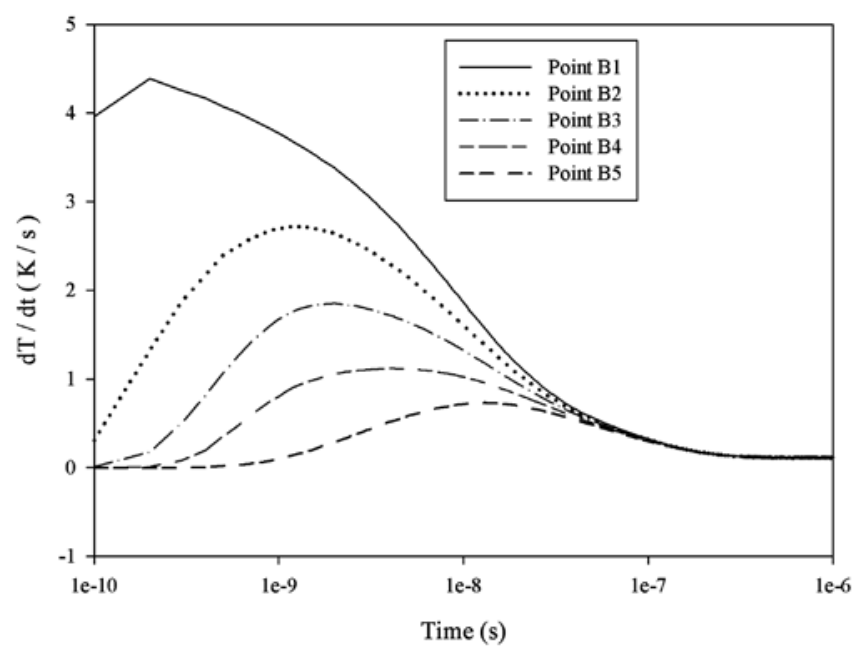

Fig. 10. Relationship between temperature variation rate and time in $\mathrm{ZnO}$ film from points $\mathrm{B} 1$ to B5 for a mesh top electrode with a $5 \mu \mathrm{m}$ electrode width and a 200 -nm-thick $\mathrm{ZnO}$ layer.

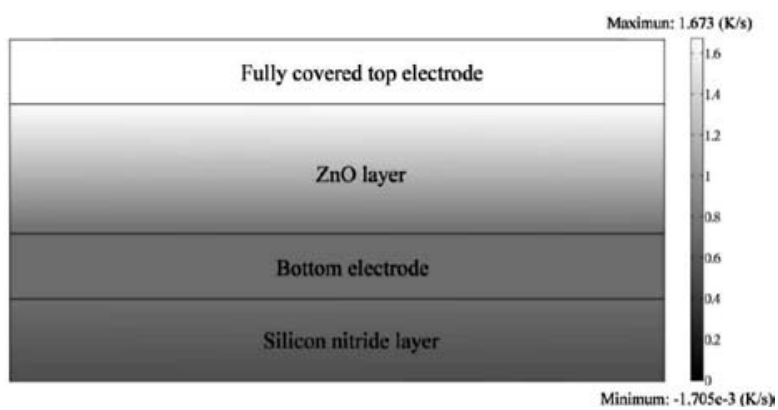

(a)

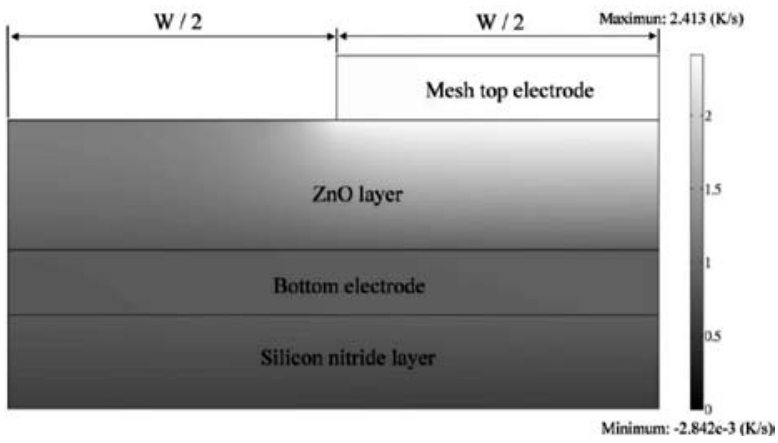

(b)

Fig. 11. Transient temperature variation field in multilayer $\mathrm{ZnO}$ pyroelectric devices: (a) fully covered electrode and (b) mesh top electrode. 
11(b) shows the transient temperature variation field at the maximum peak's time of point B5 when the mesh top electrode with $W$ of $1 \mu \mathrm{m}$ and a 200-nm-thick ZnO layer was used to fabricate the $\mathrm{ZnO}$ pyroelectric sensor. The temperature variation rate in the $\mathrm{ZnO}$ film clearly increased, which was attributed to the bare top electrode permitting the incident radiation power to directly contact the $\mathrm{ZnO}$ film. Therefore, the mesh top electrode was effective in enhancing the temperature variation rate in pyroelectric films.

When considering the 200 -nm-thick $\mathrm{ZnO}$ layer used to fabricate the $\mathrm{ZnO}$ pyroelectric devices, Fig. 12 shows the relative change in the maximum peak value of the temperature variation rate at points $\mathrm{B} 5, \mathrm{C} 5, \mathrm{D} 5$, and $\mathrm{E} 5$ in the mesh top electrode compared with that at point A5 in the fully covered electrode under various electrode width sizes. Namely, the relative change in $\mathrm{d} T / \mathrm{d} t$ can be expressed as

$$
\text { Relative change in } \mathrm{d} T / \mathrm{d} t(\%)=\left[(\mathrm{d} T / \mathrm{d} t)_{\mathrm{m}}-(\mathrm{d} T / \mathrm{d} t)_{\mathrm{f}}\right] /(\mathrm{d} T / \mathrm{d} t)_{\mathrm{f}} \times 100 \% \text {, }
$$

where $(\mathrm{d} T / \mathrm{d} t)_{\mathrm{m}}$ is the maximum peak value of the temperature variation rate at points $\mathrm{B} 5, \mathrm{C} 5, \mathrm{D} 5$, and $\mathrm{E} 5$ in the mesh top electrode, and $(\mathrm{d} T / \mathrm{d} t)_{\mathrm{f}}$ is the maximum peak value of the temperature variation rate at point $\mathrm{A} 5$ in the fully covered electrode. When $W$ was

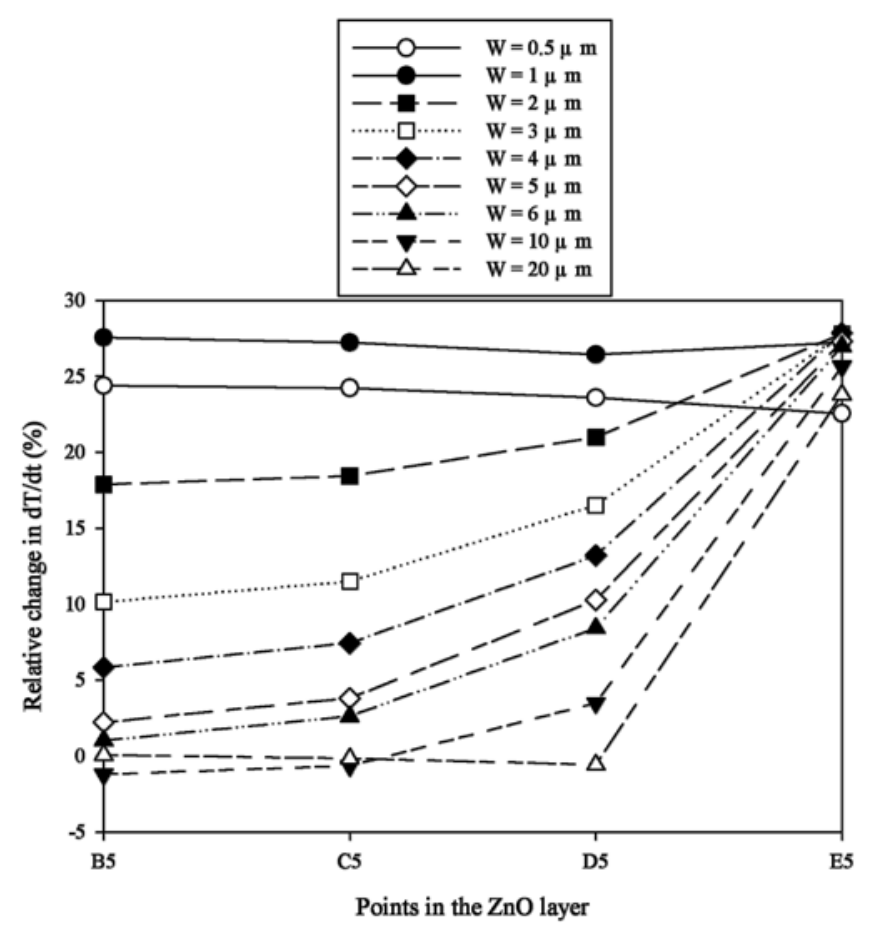

Fig. 12. Relative change in maximum peak value of temperature variation rate at points B5 to E5 in the mesh top electrode compared with that at point A5 in the fully covered electrode for a 200-nm-thick $\mathrm{ZnO}$ layer used with various electrode widths. 
larger than $10 \mu \mathrm{m}$, the maximum peak value of the temperature variation rate was no different between the mesh top electrode at points B5, C5, and D5 and the fully covered electrode at point A5, with the exception of point E5 as its point approached the bare top electrode. Therefore, attempts to improve the temperature variation rate of $\mathrm{ZnO}$ layers and responsivity of pyroelectric devices using a mesh top electrode with $W$ larger than $10 \mu \mathrm{m}$ were unsuccessful. Namely, a larger electrode width size induced the $\mathrm{ZnO}$ layer under the mesh top electrode having a lower lateral temperature gradient to absorb heat irradiation from the bare top electrode. Furthermore, the relative change in the maximum peak value of the temperature variation rate at points B5, C5, D5 and E5 in the mesh top electrode compared with that at point A5 in the fully covered electrode was about $27 \%$ when $W$ was about $1 \mu \mathrm{m}$. Hence, $W$ of $1 \mu \mathrm{m}$ was determined to be the optimum when designing the mesh top electrode for the 200-nm-thick $\mathrm{ZnO}$ film. However, when $W$ was decreased to $0.5 \mu \mathrm{m}$, the temperature variation rate declined by about $12.5 \%$ from that of the optimal $W$ of $1 \mu \mathrm{m}$ because the reduction in the bare top electrode area induced less incident radiation energy to be absorbed in the $\mathrm{ZnO}$ layer.

By increasing the thickness of the $\mathrm{ZnO}$ layer to about $600 \mathrm{~nm}$, the relative change in the maximum peak value of the temperature variation rate at points B5, C5, D5, and E5 in the mesh top electrode compared with that at point A5 in the fully covered electrode was about $17.5 \%$ when $W$ was about $2 \mu \mathrm{m}$, as shown in Fig. 13. Hence, $W$ of $2 \mu \mathrm{m}$ was

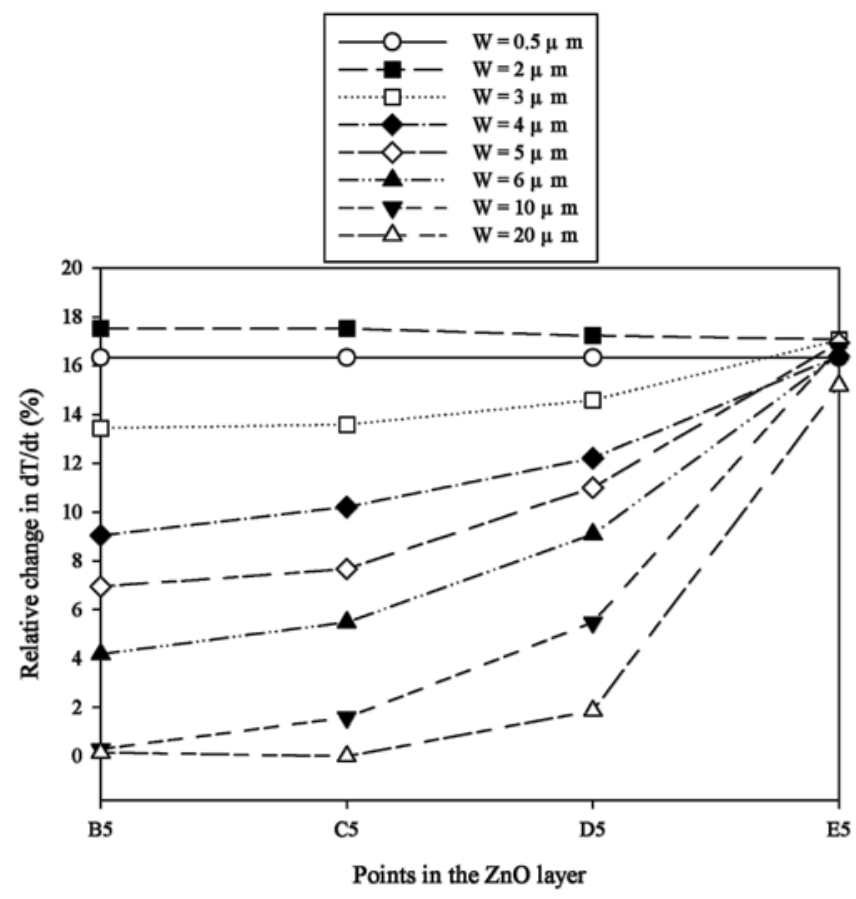

Fig. 13. Relative change in maximum peak value of temperature variation rate at points B5 to E5 in the mesh top electrode compared with that at point A5 in the fully covered electrode for a 600-nm-thick $\mathrm{ZnO}$ layer used with various electrode widths. 
the optimum when designing the mesh top electrode for the 600 -nm-thick $\mathrm{ZnO}$ film. However, decreasing $W$ to $0.5 \mu \mathrm{m}$ induced a decrease in the temperature variation rate of about $5.7 \%$ compared with that of the optimal $W$ of $2 \mu \mathrm{m}$. With the thicker $\mathrm{ZnO}$ layer of about $1200 \mathrm{~nm}$, the relative change in the maximum peak value of the temperature variation rate at points $\mathrm{B} 5, \mathrm{C} 5, \mathrm{D} 5$, and E5 in the mesh top electrode compared with that at point $\mathrm{A} 5$ in the fully covered electrode was saturated at about $12.5 \%$ when the $W$ was about $3 \mu \mathrm{m}$, as shown in Fig. 14. Hence, $W$ of $3 \mu \mathrm{m}$ was the optimum when designing the mesh top electrode for the 1200-nm-thick $\mathrm{ZnO}$ film. However, decreasing $W$ to 0.5 $\mu \mathrm{m}$ induced a decrease in the temperature variation rate of about $1.9 \%$ from that of the optimal $W$ of $3 \mu \mathrm{m}$. Hence, the effects of altering $W$ to improve the temperature variation rate were more evident with decreases in the $\mathrm{ZnO}$ film thickness. The temperature variation rate significantly declined for thinner $\mathrm{ZnO}$ films when $W$ was smaller than the optimal $W$.

Figure 15 shows the relationship between the temperature variation rate and time at point $\mathrm{B} 5$ under various $\mathrm{ZnO}$ film thicknesses and $W \mathrm{~s}$. The temperature variation rate improved by about $28.5 \%$ when $W$ of $20 \mu \mathrm{m}$ was reduced to $1 \mu \mathrm{m}$ (saturation point) when using the $200-\mathrm{nm}$-thick $\mathrm{ZnO}$ film. The temperature variation rate improved by about $16.3 \%$ when $W$ of $20 \mu \mathrm{m}$ was reduced to $2 \mu \mathrm{m}$ (saturation point) when using the

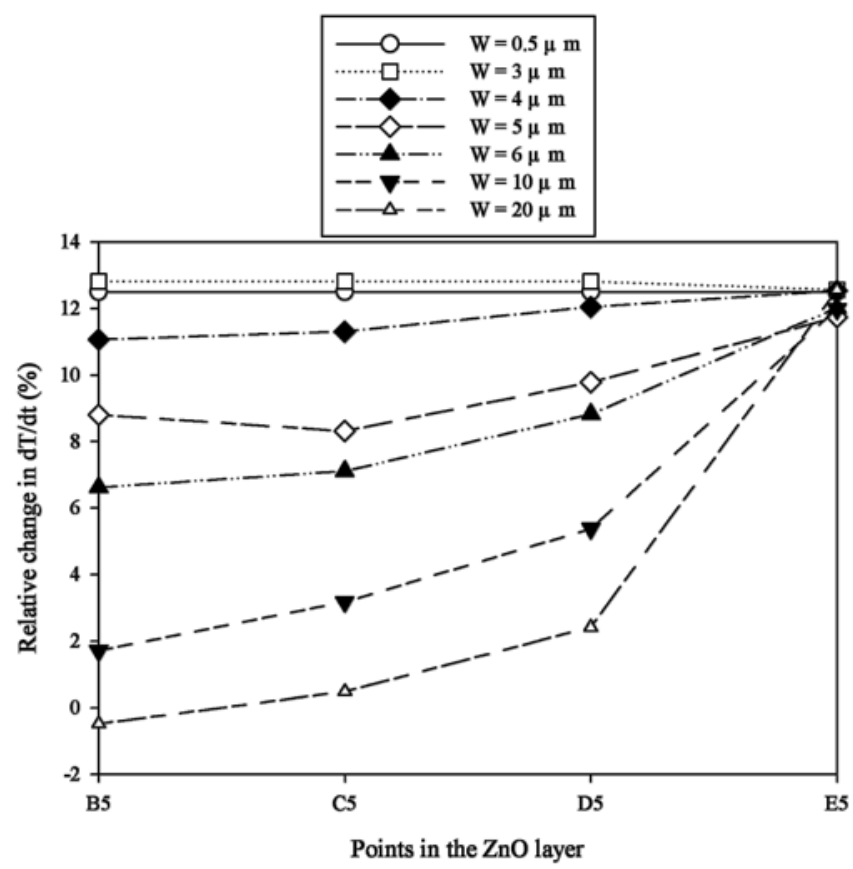

Fig. 14. Relative change in maximum peak value of temperature variation rate at points B5 to E5 in the mesh top electrode compared with that at point A5 in the fully covered electrode for a 1200-nm-thick $\mathrm{ZnO}$ layer used with various electrode widths. 


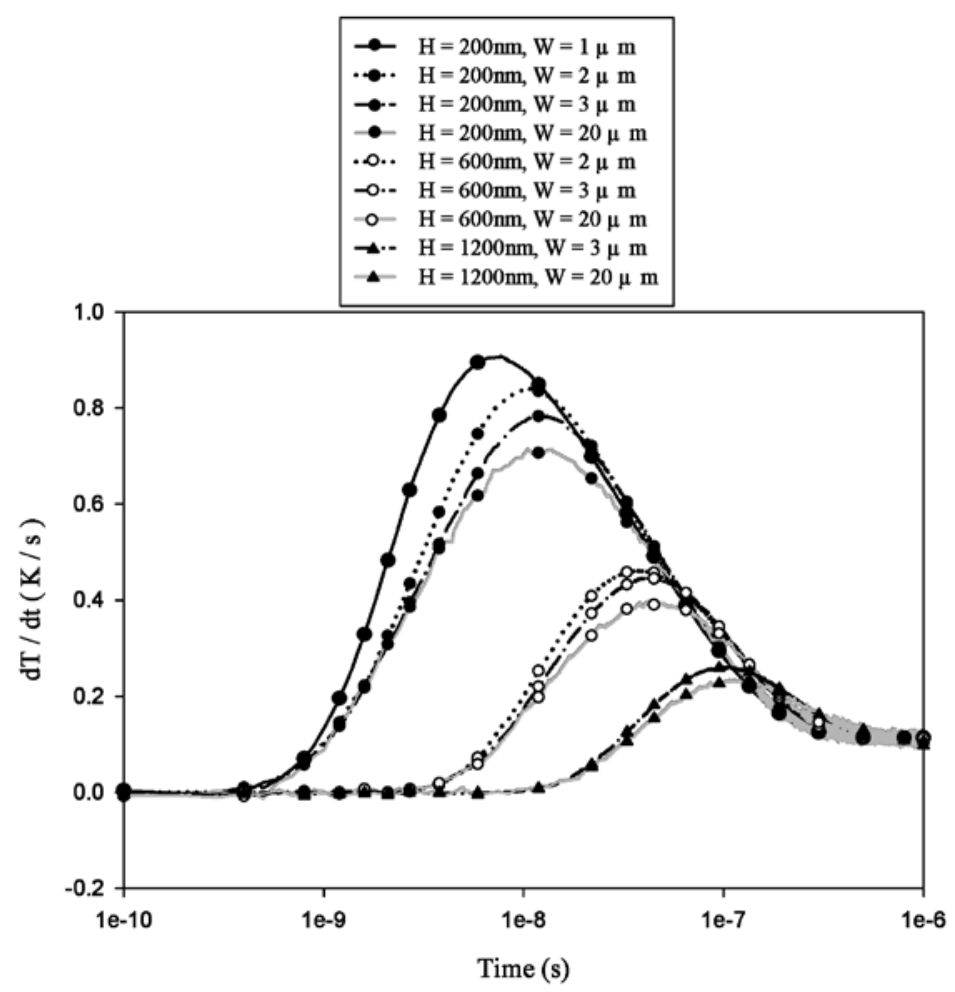

Fig. 15. Relationship between temperature variation rate $(\mathrm{d} T / \mathrm{d} t)$ and time at point B5 under various $\mathrm{ZnO}$ film thicknesses and electrode widths.

600-nm-thick $\mathrm{ZnO}$ film. The temperature variation rate improved by about $10 \%$ when $W$ of $20 \mu \mathrm{m}$ was reduced to $3 \mu \mathrm{m}$ (saturation point) when using the 1200 -nm-thick $\mathrm{ZnO}$ film. Improvements to the temperature variation rate by altering $W$ were reduced when a thicker $\mathrm{ZnO}$ film was adopted. The reason for the lack of improvement in the temperature variation rate was attributed to the thicker $\mathrm{ZnO}$ layers possessing a higher heat capacity. Hence, decreasing the $\mathrm{ZnO}$ film thickness could clearly increase the temperature variation rate and reduce the response time; then, the optimal $W$, which would further enhance the temperature variation rate, could be determined by exploring the temperature variation rate in $\mathrm{ZnO}$ layers with various thicknesses.

A transient temperature field with a chopping frequency was simulated when the multilayer $\mathrm{ZnO}$ pyroelectric devices were exposed to irradiation power with a chopping periodic time. A square waveform was produced using a programmable function generator to modulate the irradiation power with a chopping periodic time of about $2 \times 10^{-6} \mathrm{~s}$, 
as shown in Fig. 16. Figure 17 shows the temperature variation rate of the $\mathrm{ZnO}$ layer in the mesh top electrode with the optimal $W$ at point B5, compared with that in the fully covered electrode at point $\mathrm{A} 5$ under various $\mathrm{ZnO}$ film thicknesses when the incident irradiation power was modulated with a chopping frequency of about $0.5 \mathrm{MHz}$. The temperature variation rate in the 200 -nm-thick $\mathrm{ZnO}$ film was clearly larger than those in the films of 600 and $1200 \mathrm{~nm}$ thicknesses. Altering $W$ to improve the temperature variation rate had a clearer effect for thinner pyroelectric films.

An experimental setup was used to verify the above analytical results. The voltage responsivity is proportional to the temperature variation rate in the $\mathrm{ZnO}$ layer. Therefore, the IR laser beam was chopped and molded as a square wave with a modulated frequency to obtain a temperature variation in the $\mathrm{ZnO}$ layer by a programmable function generator. In the present study, a two-step sputtering process was used to deposit a $1.2-\mu \mathrm{m}$-thick $\mathrm{ZnO}$ film. Figure 18 shows the X-ray diffraction pattern for the $\mathrm{ZnO}$ film deposited by the proposed two-step sputtering, which was observed to possess the growth characteristic of strongly preferred orientation toward the c-axis. The fabricated $\mathrm{ZnO}$ pyroelectric sensors with $W_{\mathrm{s}}$ of 30,100 , and $500 \mu \mathrm{m}$ are shown in Fig. 19. Figure 20 shows the voltage responsivities $(R v)$ of the present $\mathrm{ZnO}$ pyroelectric sensors with $W \mathrm{~s}$ of 30,100 , and $500 \mu \mathrm{m}$. $R v$ is defined as the ratio of the output voltage of the sensor to the input power of the incident heat source. At identical total top electrode areas about $12.15 \mathrm{~mm}^{2}$ for the present $\mathrm{ZnO}$ pyroelectric sensors with $W \mathrm{~s}$ of 30,100 , and $500 \mu \mathrm{m}$, the $\mathrm{ZnO}$ pyroelectric sensor with $W$ of $30 \mu \mathrm{m}$ had a larger voltage responsivity of about $4.519 \mathrm{~V} / \mathrm{W}$ at the frequency of $100 \mathrm{~Hz}$ than that with $W$ of $100 \mu \mathrm{m}$ (about $4.281 \mathrm{~V} / \mathrm{W}$ ) and $500 \mu \mathrm{m}$ (about $3.628 \mathrm{~V} / \mathrm{W}$ ). Moreover, the difference in the voltage responsivity among various $W \mathrm{~s}$ decreased when increasing frequency. A larger $W$ was helpless to promote the temperature variation rate and the voltage responsivity. Therefore, these experimental results were sufficient to verify the simulation results, and $W$ was a critical parameter for designing a pyroelectric sensor.

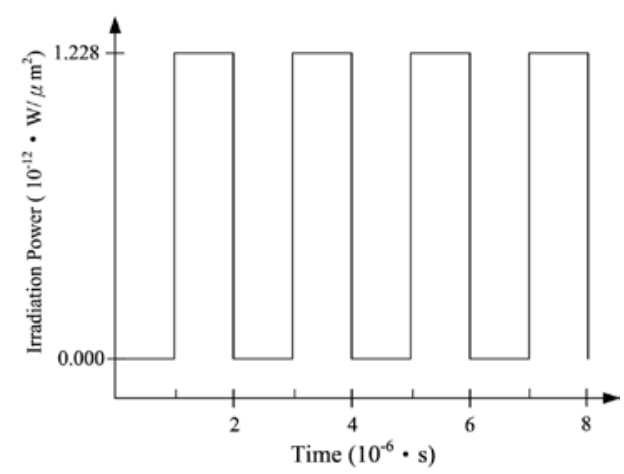

Fig. 16. Square waveform used to modulate the irradiation power using a programmable function generator. 

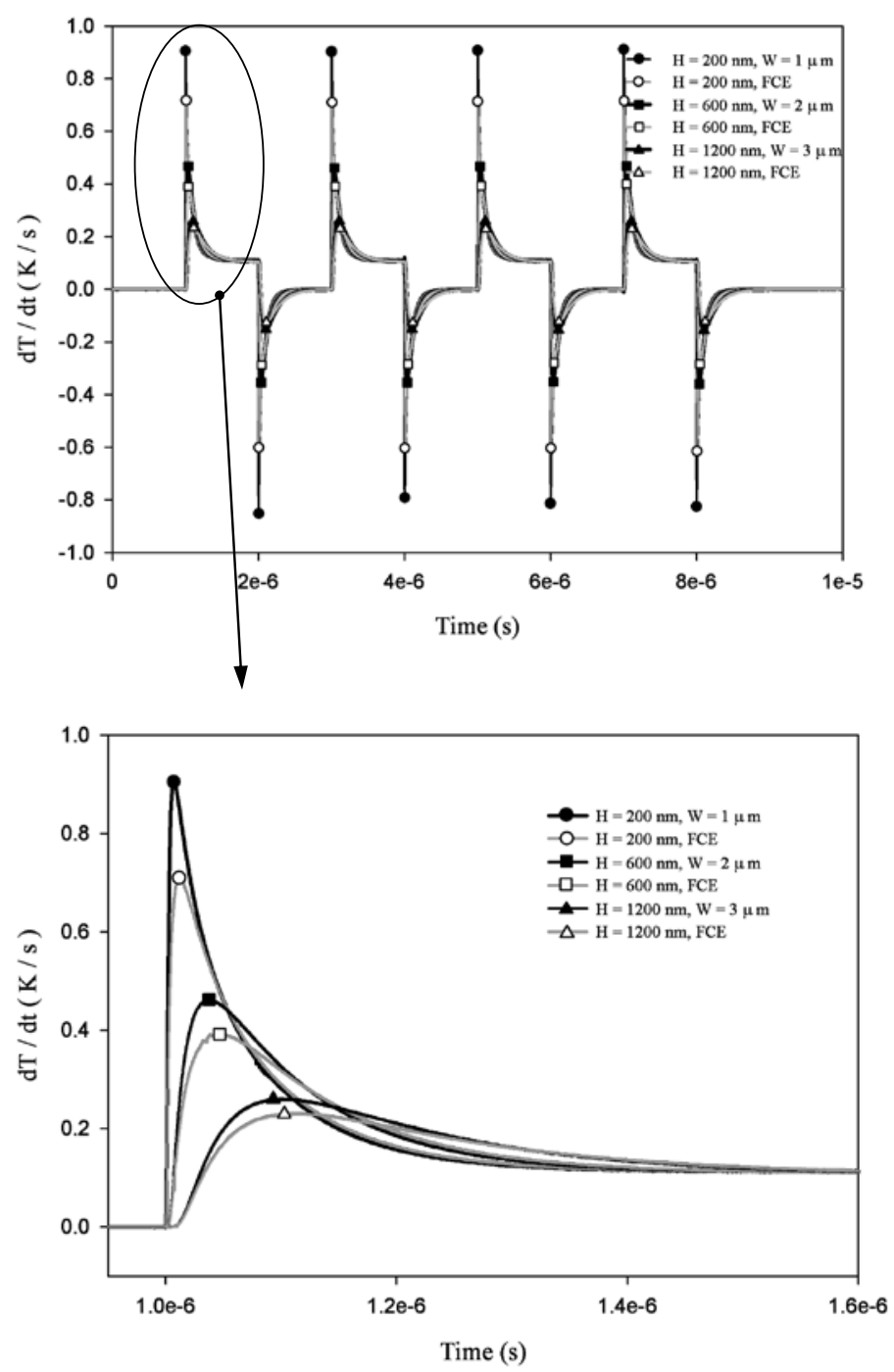

Fig. 17. Temperature variation rate of $\mathrm{ZnO}$ layer in the mesh top electrode with the optimal electrode width at point B5 compared with that in the fully covered electrode (FCE) at point A5 for various $\mathrm{ZnO}$ film thicknesses upon the incident irradiation power modulated with a chopping frequency of about $0.5 \mathrm{MHz}$. 


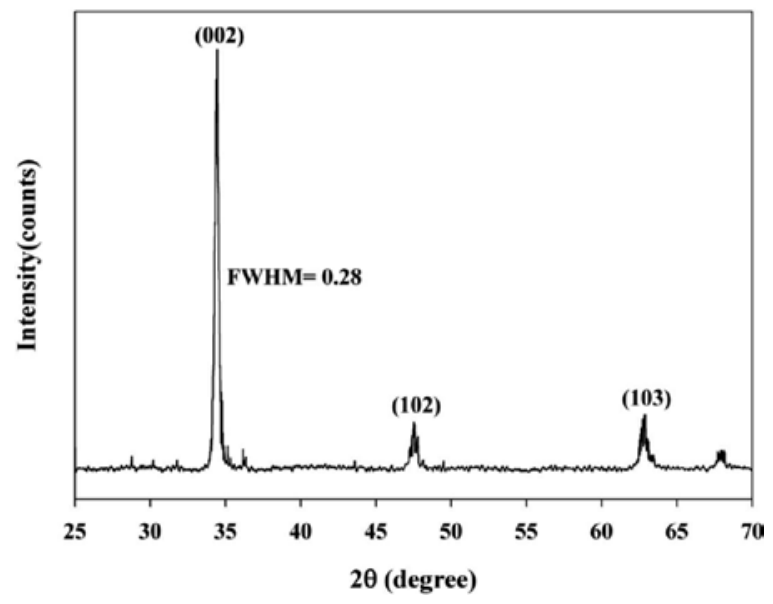

Fig. 18. XRD pattern of the $\mathrm{ZnO}$ film deposited by the two-step sputtering process.

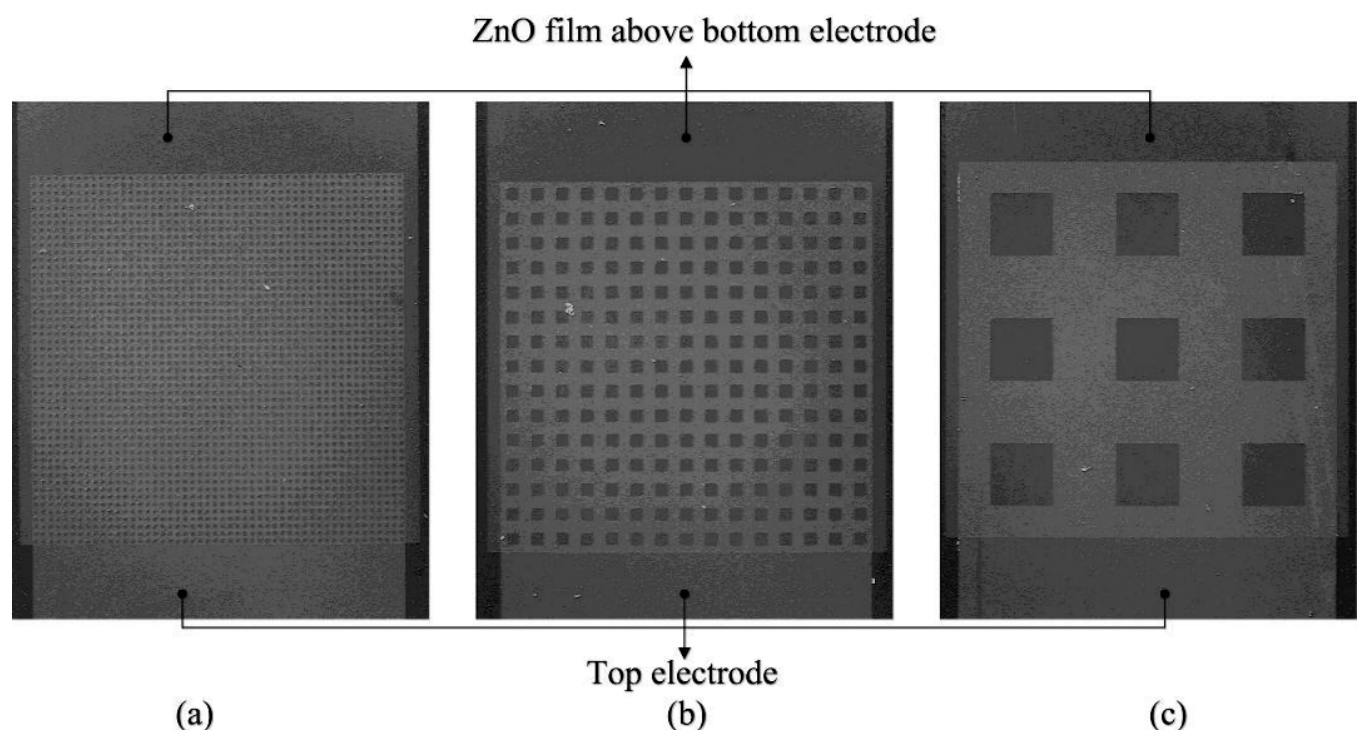

Fig. 19. The fabricated $\mathrm{ZnO}$ pyroelectric sensors with various $W \mathrm{~s}$ : (a) 30 , (b) 100 , and (c) $500 \mu \mathrm{m}$. 


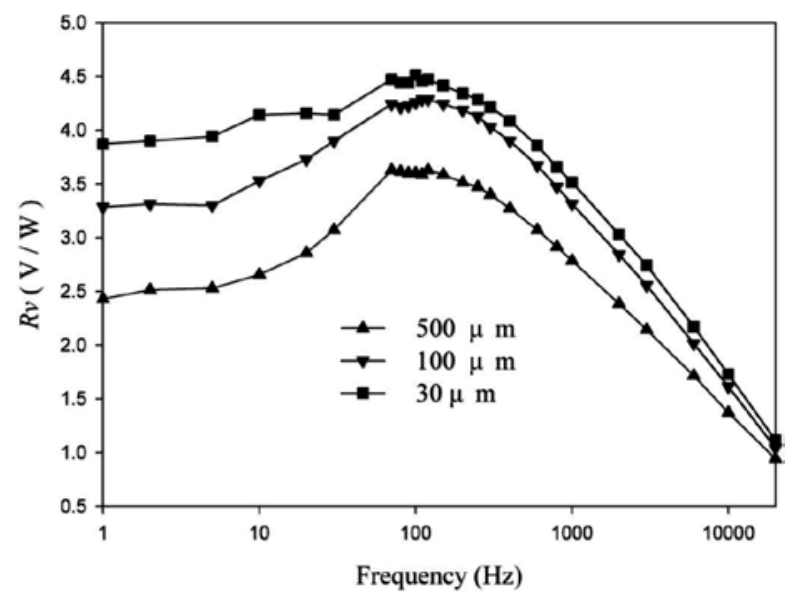

Fig. 20. Voltage responsivities $(R v)$ of the $\mathrm{ZnO}$ pyroelectric sensors with various $W \mathrm{~s}$.

\section{Conclusions}

A finite element model is used to explore the temperature variation rate in multilayer $\mathrm{ZnO}$ pyroelectric sensors with the mesh top electrode compared with the fully covered top electrode. The partially covered top electrode was remarkable in improving the responsivity of pyroelectric devices. An optimal $W$ was obtained by exploring the temperature variation rate in $\mathrm{ZnO}$ layers of various thicknesses. Decreasing the $\mathrm{ZnO}$ film thickness was beneficial in improving the temperature variation rate and optimal $W$, as determined, and further enhanced the temperature variation rate. Improvements in the temperature variation rate by altering $W$ were more obvious when the $\mathrm{ZnO}$ film thickness was reduced. Moreover, the temperature variation rate significantly declined for thinner $\mathrm{ZnO}$ films when $W$ was smaller than the optimal value. In addition, an experimental result was successful to verify the simulation results, and $W$ was a critical parameter for designing a pyroelectric sensor.

\section{Acknowledgments}

The authors are thankful for the financial support from the National Science Council of Taiwan through Grant No. NSC 99-2628-E-150-003, and the experimental support from the Common Lab for Micro/Nano Science and Technology at the National Formosa University. 


\section{References}

1 R. W. Whatmore: Rep. Prog. Phys. 49 (1986) 1335.

2 S. G. Porter: Ferroelectrics 33 (1980) 193.

3 C. C. Chang and C. S. Tang: Sens. Actuators, A 65 (1998) 171.

4 A. Cuadrasa, M. Gasulla and V. Ferrari: Sens. Actuators, A 158 (2010) 132.

5 C. S. Wei, Y. Y. Lin, Y. C. Hu, C. W. Wu, C. K. Shih, C. T. Huang and S. H. Chang: Sens. Actuators, A 128 (2006) 18.

6 C. C. Hsiao, Y. C. Hu and R. C. Chang: Sens. Mater. 22 (2010) 417.

7 V. Norkus, A. Schulze, Y. Querner and G. Gerlach: Procedia Eng. 5 (2010) 944.

8 L. Li, L. Zhang, X. Yao and B. Li: Ceram. Int. 30 (2004) 1847.

9 C. C. Hsiao, K. Y. Huang and Y. C. Hu: Sensors 8 (2008) 185.

10 S. H. Park, B. C. Seo, H. D. Park and G. Yoon: Jpn. J. Appl. Phys. 39 (2000) 4115. 